\title{
A gestão do SUS e as práticas de monitoramento e avaliação: possibilidades e desafios para a construção de uma agenda estratégica
}

\author{
SUS management and monitoring and evaluation practices: \\ possibilities and challenges for building a strategic agenda
}

\author{
André Luis Bonifácio de Carvalho ${ }^{1}$ \\ Maria de Fátima Souza ${ }^{2}$ \\ Helena Eri Shimizu ${ }^{2}$ \\ Isabel Maria Vilas Boas Senra ${ }^{1}$ \\ Kátia Cristina de Oliveira ${ }^{1}$
}

\footnotetext{
${ }^{1}$ Departamento de Monitoramento e Avaliação da Gestão do SUS, Secretaria de Gestão Estratégica e Participativa, Ministério da Saúde. Esplanada dos Ministérios Bloco G Asa Sul. 70058-900 Brasília DF.

andre.bonifacio@saude.gov.br ${ }^{2}$ Núcleo de Estudos em Saúde Publica,

Universidade de Brasília
}

\begin{abstract}
This paper deals with the challenges involved in institutionalizing Monitoring and Evaluation practices within the scope of management of the Brazilian Unified Health System (SUS), based on the creation of a strategic agenda. This is structured around actions by the Federal Administration, from the perspective of defining the scope of the federative relationship. A summarized literature review is thus presented, placing into context the current SUS management process, which is based on the Pact for Health as well as theoretical and operational aspects inherent to monitoring and evaluation practices. The approach related to the strategic agenda highlights the creation of mechanisms that underpin the political and institutional decision, namely the creation of financial incentives and the development of technical mechanisms. These include organizational strategies for qualifying management teams and conducting studies and research and the dissemination of strategic information. These processes are based on the concept of providing for the management specificities in the different spheres of management based on a broad cooperation process involving teaching and research institutions and international organizations as well as the managers themselves.

Key words Health management, Health monitoring, Health evaluation, Strategic and participative management
\end{abstract}

Resumo O presente artigo trata dos desafios de se institucionalizar as práticas de Monitoramento e Avaliação no âmbito da Gestão do Sistema Único de Saúde, tendo como base a construção de uma agenda estratégica, organizada a partir das ações do Gestor Federal, na perspectiva de qualificar o espaço da relação federativa. Para tanto é apresentada uma revisão sumária da literatura, contextualizando o atual processo da gestão do SUS, que tem como base o Pacto pela Saúde, como também aspectos teóricos e operacionais inerentes às práticas do monitoramento e avaliação. A abordagem relacionada à agenda estratégica destaca a criação de mecanismos que articulam a decisão políticoinstitucional, a criação de incentivos financeiros, o desenvolvimento de mecanismos técnicos; estratégias organizacionais de qualificação das equipes gestores; a realização de estudos e pesquisa e a divulgação de informações estratégicas, processos estes pautados pela concepção de atender as especificidades da gestão nas suas diferentes esferas e tendo como base um amplo processo de cooperação envolvendo, além dos gestores, as instituições de ensino e pesquisa e as organizações internacionais.

Palavras-Chave Gestão em saúde, Monitoramento em saúde, Avaliação em saúde, Gestão estratégica e participativa 


\section{Gestão do SUS e sua interface com processos e práticas de monitoramento e avaliação: Fazendo o caminho na caminhada}

O processo de institucionalização do Sistema Único de Saúde (SUS), como política de caráter federativo e intergovernamental, gradualmente vem estabelecendo as novas competências e responsabilidades governamentais, que implicam constantes negociações e pactos intergestores; em meio a inovações conceituais, logísticas, tecnológicas e instrumentais, que influem cotidianamente no exercício da gestão setorial, muitas vezes em condições heterogêneas, diversas e plurais.

Segundo Miranda ${ }^{1}$, a gestão governamental em saúde é constituída a partir de uma práxis social de âmbito institucional, possuindo uma natureza complexa e um caráter polivalente (ético, político, estratégico, técnico, administrativo). Seus modos de ser estão conformados em uma dimensão política, a partir da intermediação de interesses distintos, das interações de poder e de margens de autonomia; constituídos a partir de ações intencionais ou comportamentos regrados de atores sociais; expressos em distintas racionalidades da ação; desencadeados a partir de decisões (formais e informais); e consubstanciados em combinações tecnológicas, métodos, técnicas, instrumentos e atividades intermediárias nos sistemas institucionalizados de ação.

Miranda ${ }^{1}$, afirma que o propósito primordial da gestão em saúde é a produção de decisões, que desencadeiam o processo de intermediação e implementação das políticas. Há muita complexidade e subjetividade envolvida nesses processos de gestão. Os sistemas de direção, assim como o processo e os arranjos para a tomada de decisões, envolvem motivações, interesses, racionalidades imbricadas, intuição (e experiência intuitiva), conhecimentos, capacidades cognitivas, habilidades, qualidade estratégica, controle de capitais e recursos (tempo, informações, tecnologias, financiamento). Enfim, características e qualidades de pessoas (sujeitos, agentes, atores institucionais) e não somente de sistemas ou serviços.

Santos e Andrade ${ }^{2}$ destacam que o SUS é o exemplo mais acabado de federalismo cooperativo, onde os interesses são comuns e indissociáveis e devem ser harmonizados em nome dos interesses local, regional, estadual e nacional. No federalismo (no SUS), todos são cotitulares dos interesses (da saúde) e todos devem manter garantida sua autonomia (direção única).
No caso do SUS, além do desenvolvimento de toda uma logística e tecnologia de regulação baseada em necessidades prioritárias, vulnerabilidades e riscos, há a necessidade de mediação política e definição de diretrizes e de critérios a partir dos espaços de participação e de controle social.

No contexto atual o processo de gestão do SUS tem no Pacto pela Saúde a estratégia para um novo pacto federativo, articulado em três dimensões, o Pacto pela Vida, o Pacto em Defesa do SUS e o Pacto de Gestão, conforme o Quadro 1, o qual apresenta de forma sucinta a descrição dos componentes do Pacto pela Saúde e os instrumentos/processos para a sua implantação.

A recente construção do Pacto pela Saúde e seu aprimoramento com a promulgação do Decreto 7508 , de 28 de junho de 2011, que regulamenta aspectos da Lei 8080/90, tem como fundamento principal o respeito aos princípios constitucionais do SUS, com ênfase nas necessidades de saúde da população, o que implica o exercício simultâneo da definição de prioridades articuladas e integradas buscando a melhoria do acesso a ações e serviços de saúde, o fortalecimento do planejamento regional com a consequente definição das redes de atenção nas regiões de saúde, o aprimoramento dos mecanismos de governança e a qualificação dos processos de pacto tripartite.

Estes aspectos inerentes ao processo de implantação do novo pacto federativo precisam ser considerados para a construção de métodos que auxiliem os gestores a qualificar os processos e as práticas de monitoramento e avaliação no âmbito da gestão do SUS, influindo de maneira positiva na tomada de decisão.

Paim $^{3}$ afirma que diversas iniciativas para a avaliação em saúde no Brasil vêm sendo desenvolvidas de forma progressiva, nas últimas três décadas, e ressalta que na presente pode-se afirmar que o interesse pela avaliação não se restringe ao âmbito acadêmico, e que o próprio $\mathrm{Mi}$ nistério da Saúde tem encomendado um conjunto de estudos nessa perspectiva, não só por exigência de financiamento externo, mas devido a uma crescente consciência de responsabilização entre seus técnicos e dirigentes.

Furtado e Laperrièrre ${ }^{4}$, citando Figueiredo e Tanaka, afirmam que, no Brasil, a avaliação desperta crescente interesse a partir dos anos 1990, quando a ampliação do número de iniciativas ligadas às políticas públicas sociais aumenta, no contexto da Constituição de 1998, que amplia os direitos civis dos cidadãos brasileiros. O Sistema Único de Saúde, nesse bojo, vem ampliar a importância econômica e política de serviços e pro- 
Quadro 1. Pacto pela Saúde: componentes, aspectos e instrumentos.

\begin{tabular}{|c|c|c|}
\hline Componentes & Aspectos & Instrumentos/Processos \\
\hline $\begin{array}{l}\text { Pacto pela } \\
\text { Vida }\end{array}$ & $\begin{array}{l}\text { O Pacto pela Vida está constituído por um conjunto de } \\
\text { compromissos sanitários, expressos em objetivos de processos e } \\
\text { resultados e derivados da análise da situação de saúde do País e das } \\
\text { prioridades definidas pelos governos federal, estaduais e } \\
\text { municipais. Significa uma ação prioritária no campo da Saúde que } \\
\text { deverá ser executada com foco em resultados e com a explicitação } \\
\text { inequívoca dos compromissos orçamentários e financeiros para o } \\
\text { alcance desses resultados. }\end{array}$ & $\begin{array}{l}\text { Unificação dos pactos de } \\
\text { indicadores existentes } \\
\text { normatizada na Portaria GM } \\
\text { no91 de } 10 \text { de janeiro de } 2007 \text { e } \\
\text { atualizada em 2008, pela Portaria } \\
\text { GM no } 325 \text {, ficando estabelecidas } \\
\text { nacionalmente, nesta última, } 11 \\
\text { prioridades, com } 41 \text { objetivos e } \\
43 \text { indicadores. }\end{array}$ \\
\hline $\begin{array}{l}\text { Pacto em } \\
\text { Defesa do SUS }\end{array}$ & $\begin{array}{l}\text { O Pacto em Defesa do SUS envolve ações concretas e articuladas } \\
\text { pelas três instâncias federativas, no sentido de reforçar o SUS, } \\
\text { como Política de Estado mais do que política de governos; e de } \\
\text { defender, vigorosamente, os princípios basilares dessa política } \\
\text { pública, inscritos na Constituição Federal. A concretização do } \\
\text { Pacto pela Saúde passa por um movimento de repolitização da } \\
\text { Saúde, com uma clara estratégia de mobilização social com } \\
\text { envolvimento conjunto da sociedade brasileira, extrapolando os } \\
\text { limites do setor e vinculada ao processo de instituição da Saúde } \\
\text { como direito de cidadania, tendo o financiamento público como } \\
\text { um dos pontos centrais. }\end{array}$ & $\begin{array}{l}\text { Implementar um Projeto de } \\
\text { Mobilização Social com a } \\
\text { finalidade de discutir a Saúde } \\
\text { como direito de cidadania e o } \\
\text { SUS como sistema público } \\
\text { universal garantidor desses } \\
\text { direitos, a exemplo da Caravana } \\
\text { em Defesa do SUS, proposta pelo } \\
\text { Conselho Nacional de Saúde, } \\
\text { como uma de suas agendas } \\
\text { prioritárias; } \\
\text { Alcançar, no curto prazo, a } \\
\text { regulamentação da Emenda } \\
\text { Constitucional no 29, pelo } \\
\text { Congresso Nacional; } \\
\text { Divulgar a Carta dos Direitos dos } \\
\text { usuários do SUS }\end{array}$ \\
\hline $\begin{array}{l}\text { Pacto de } \\
\text { Gestão }\end{array}$ & $\begin{array}{l}\text { O Pacto de Gestão estabelece as responsabilidades claras de cada } \\
\text { ente federado, de forma a diminuir as competências concorrentes } \\
\text { e a tornar mais claro quem deve fazer o quê, contribuindo, assim, } \\
\text { para o fortalecimento da gestão compartilhada e solidária do SUS. } \\
\text { Radicaliza a descentralização, reforça a territorialização da saúde } \\
\text { como base para organização dos sistemas, instituindo colegiados } \\
\text { de gestão regional. Reitera a importância da participação e do } \\
\text { controle social com o compromisso de apoio à sua qualificação e } \\
\text { explicita as diretrizes para o sistema de financiamento público } \\
\text { tripartite, buscando critérios de alocação equitativa dos recursos; } \\
\text { reforça os mecanismos de transferência fundo a fundo entre } \\
\text { gestores, através da instituição de cinco grandes blocos de } \\
\text { financiamento federal: atenção básica, média e alta complexidade } \\
\text { da assistência à saúde, vigilância em saúde, assistência } \\
\text { farmacêutica e gestão do SUS. Por fim, estabelece as diretrizes } \\
\text { para a gestão do SUS, com ênfase na Descentralização; } \\
\text { Regionalização; Financiamento; Programação Pactuada e } \\
\text { Integrada; Regulação; Participação e Controle Social; } \\
\text { Planejamento; Gestão do Trabalho e Educação na Saúde. }\end{array}$ & $\begin{array}{l}\text { Termo de Compromisso de } \\
\text { Gestão e seus anexos Portaria } \\
\text { GM no 699, de } 30 \text { de março de } \\
2006\end{array}$ \\
\hline
\end{tabular}

Fonte: Portaria GM 399 de 22de Fevereiro de 2006 e Portaria GM 699 de 30 de março de 2006.

gramas de saúde. Configurando-se como o grande projeto social do pais, as ações do SUS tornam-se alvo de questionamentos sobre sua eficiência e efetividade, estimulando o surgimento de demandas por avaliações sistemáticas.
Em recente publicação, Figueiró et al. ${ }^{5}$ reforçam os aspectos destacados por Paim ${ }^{3}$ e afirmam que no campo das ações políticas, e especificamente no das políticas de saúde, a avaliação vem ganhando uma centralidade nas organiza- 
ções. Entre os fatores envolvidos no interesse crescente em avaliação de políticas, programas e serviços de saúde no Brasil podem-se apontar: as mudanças nos procedimentos legais e administrativos na gestão do Sistema Único de Saúde (SUS), como processo da descentralização das responsabilidades, ações e recursos; a maior complexidade do perfil epidemiológico do país com distintos problemas e necessidades, requerendo novas abordagens e incorporando novas tecnologias; as exigências cada vez maiores do controle de gastos em saúde e, com mais frequência e contundência, a cobrança dos organismos financiadores externos.

Porém, segundo Felisberto ${ }^{6}$, em que pese às recentes iniciativas do Ministério da Saúde, os avanços, na maior parte dos casos, constituíramse em iniciativas pontuais, mediante pesquisas específicas, estudos de casos ou produção regular de dados sem a equivalente regularidade de análise dos mesmos orientada para as necessidades da gestão. O próprio Ministério da Saúde ${ }^{7}$ identifica alguns dos limites dessas iniciativas: 1) a ausência ou insuficiência da explicitação das diretrizes políticas e da definição estratégica que orientam a avaliação; 2) a fragmentação e a decorrente diversidade de orientações que presidem os processos de avaliação, impedindo que possam ser úteis a uma ação coordenada; 3) a ênfase em variáveis relacionadas a processo; $e, 4)$ avaliações de resultados pontuais elou espacialmente restritas.

Estes pontos são reforçados pelo próprio Ministério da Saúde ${ }^{7}$, ao afirmar que no Brasil, a avaliação em saúde apresenta-se, ainda, por intermédio de processos incipientes, pouco incorporados às práticas, possuindo, quase sempre, um caráter mais prescritivo e burocrático. Não faz parte da cultura institucional, aparecendo de maneira pouco ordenada e sistematizada, e nem sempre contribuindo com o processo decisório e com a formação dos profissionais, necessitando, portanto, de investimentos de ordem técnica e política por parte da administração pública setorial.

Em que pese os pontos de fragilidade inerentes à própria dinâmica de construção e de desenvolvimento de iniciativas no âmbito da gestão, podemos afirmar que temos muitos avanços, mas também é patente que os inúmeros esforços ainda ocorrem de forma fragmentada, frequentemente restritos aos objetivos específicos e aos processos de gestão de alguns programas, mas raramente utilizados para redesenhar suas estratégias e formas de operação. Desses esforços resultaram muitos documentos, muita informação e uma baixa capacidade de produzir uma visão integrada do desempenho do sistema de saúde, como também do processo de gestão no âmbito do SUS.

Neste artigo, apresentamos as iniciativas recentes do Ministério da Saúde por meio da Secretaria de Gestão Estratégica e Participativa (SGEP/ MS) no período de 2007 a 2010, tendo como base elementos operacionais e teórico-conceituais em torno da qualificação dos processos e da prática do monitoramento e da avaliação da gestão do SUS, utilizando como móvel a estratégia de indução/cooperação por parte do gestor federal.

\section{Monitoramento e Avaliação da Gestão do SUS, abordagem conceitual aspectos da institucionalização através da indução do gestor federal: Navegar é Preciso}

A criação da Secretaria de Gestão Estratégica e Participativa (SGEP) representou uma inovação no processo de cooperação do Ministério da Saúde junto às gestões estaduais e municipais, bem como com as instâncias de participação e controle social, particularmente por sua responsabilidade em qualificar e aperfeiçoar a implementação das práticas de gestão estratégica e participativa nas três esferas de gestão do SUS.

As ações da SGEP estão articuladas através dos quatro departamentos que a compõem, ou seja, Departamento de Gestão Participativa (Dagep), Departamento Nacional de Auditoria do SUS (Denasus), Departamento de Monitoramento e Avaliação da Gestão do SUS (Demags) e Departamento Nacional de Ouvidoria (Doges).

No que tange as ações do DEMAGS, fruto de um intenso debate promovido pelo Ministério da Saúde, entre 2003 e 2005, envolvendo diversos órgãos, evidenciou-se a necessidade de uma estrutura que assumisse as tarefas de articulação, apoio e difusão das ações de monitoramento e avaliação que se encontram em curso nas diversas Secretarias do Ministério da Saúde e demais unidades, bem como nas outras esferas do SUS, motivando, desta forma, a sua estruturação no ano de 2005 e sua criação em 2006.

Esta ação do Ministério da Saúde destaca-se como mais uma iniciativa rumo ao processo de institucionalização da avaliação em saúde, porém Felisberto", afirma que tentativas de "institucionalizar" a avaliação no SUS a partir da criação de "Departamentos" em Secretarias do Ministério da Saúde ou em outras instâncias organizacionais, parecem, por si só, não lograrem êxito. Em que pese o autor não desconsiderar a importância da iniciativa o mesmo acredita ser condição essencial 
ter a avaliação como prática "introjetada" ou "vivenciada" pelos atores institucionais.

Destacamos neste ponto a influência da abordagem trazida pela "quarta geração" da avalição, ao trabalho desenvolvido pelo DEMAGS, Dubois et al. ${ }^{8}$ afirmam que ela se tornou um instrumento de negociação e de fortalecimento do poder (empowerment). Nessa nova abordagem, a avaliação não é um campo reservado aos especialistas. Pelo contrário, é aberta a todos os participantes da ação social, inclusive aos grupos marginalizados e pouco favorecidos da sociedade.

Por fim, utilizando a reflexão de Habermans, Dubois et al. ${ }^{8}$ asseveram que ela favorece o dialogo, a reflexão e a compreensão mútua dos diferentes atores.

Estas considerações são extremamente pertinentes tendo em vista as responsabilidades atribuídas a SGEP, não apenas no que tange aos aspectos da avaliação, mas à sua interface com as ações da auditoria, da ouvidoria e da gestão participativa, onde os aspectos inerentes ao controle social e à participação popular são fundamentais para a qualificação da gestão do SUS.

Porém, antes de uma aproximação maior aos aspectos inerentes à discussão da institucionalização das práticas e processos de monitoramento e avaliação, cabe destacar que as preocupações com estas ações antecedem a criação do SUS, com diversos movimentos visando o estabelecimento de conceitos, metodologias e práticas.

No que concerne aos aspectos do monitoramento, Garcia ${ }^{9}$ afirma ser um processo sistemático e contínuo que produz informações sintéticas em tempo eficaz, permitindo uma rápida avaliação situacional, propiciando uma intervenção oportuna. Pode ser entendido como uma ação que permite a observação, a medição e a avaliação contínua de um processo ou fenômeno.

Santos et al. ${ }^{10}$, citando Poister, afirmam que o monitoramento e a avaliação são ferramentas críticas para a gestão. O monitoramento é fundamental para o acompanhamento rotineiro de informações prioritárias, tanto para o processo de implementação de um programa, isto é, para o acompanhamento de seu desempenho operacional, como para seu desempenho finalístico.

Sendo assim é patente que o monitoramento no âmbito da gestão pública não poderá ser eficaz se a equipe gestora não conhecer de maneira contínua e objetiva os sinais vitais dos processos que conduzem, como também da(s) situação(ões) na(s) qual(is) intervém.

A avaliação por sua vez traz a visão de julgamento, prática de intervenções que auxiliam na tomada de decisão, ação capaz de subsidiar mudanças na construção e/ou na implementação de programas, projetos ou políticas de saúde. Cabe destacar que, segundo Figueiró et al. ${ }^{5}$, como uma área do conhecimento considerada emergente e jovem, a avaliação apresenta consensos e dissensos nas suas definições, referenciais teóricos e modelos.

Para Garcia ${ }^{9}$, avaliar não significa apenas medir, mas antes de mais nada julgar a partir de um referencial de valores. É estabelecer, a partir de uma percepção intersubjetiva e valorativa, com base nas melhores medições objetivas, o confronto entre a situação atual com a ideal, o possível afastamento dos objetivos propostos, das metas a alcançar, de maneira a permitir uma constante e rápida correção de rumos, com economia de esforços (recursos) e de tempo.

Segundo Contandriopoulos ${ }^{11}$, avaliar consiste fundamentalmente em aplicar um julgamento de valor a uma intervenção através de um dispositivo capaz de fornecer informações cientificamente válidas e socialmente legítimas sobre ela ou qualquer um dos seus componentes, permitindo aos diferentes atores envolvidos, que podem ter campos de julgamento diferentes, se posicionarem e construírem (individual ou coletivamente) um julgamento capaz de ser traduzido em ação.

Minayo $^{12}$ destaca a avaliação como técnica e estratégia investigativa, é um processo sistemático de fazer perguntas sobre o mérito e a relevância de determinado assunto, proposta ou programa. Um processo de avaliação de programas e projetos sociais geralmente tem como sentido mais nobre, fortalecer o movimento de transformação da sociedade em prol da cidadania e dos direitos humanos.

Segundo $\mathrm{Pisco}^{13}$, a avaliação é, pois, um poderoso instrumento de mudança que não deve ser visto como uma ameaça, mas sim como um incentivo para que os diferentes serviços de saúde cumpram padrões mínimos de qualidade.

Brito e Jesus ${ }^{14}$, citando Silva, destacam que as novas responsabilidades com a gestão do sistema de saúde requerem a incorporação da avaliação como componente do processo de planejamento, como atividade capaz de subsidiar as tomadas de decisões e como elemento auxiliar nas iniciativas voltadas para a mudança do modelo assistencial. Sua adoção como uma atividade sistemática ao interior da gestão municipal, e completamos estadual e federal, pode ser considerado um dos indicadores de modificação do modelo assistencial. 
Hartz e Vieira ${ }^{15}$, expressam a institucionalização da avaliação com o sentido de integrá-la em um sistema organizacional que seja capaz de influenciar o seu comportamento, ou seja, um modelo orientado para a ação, ligando necessariamente as atividades analíticas às de gestão das intervenções programáticas.

Takeda e Yves ${ }^{16}$ afirmam que Avaliar é uma responsabilidade e as instituições não podem se furtar em exercê-la. Institucionalizar a avaliação significa incorporá-la ao sistema, possibilitando monitorar a capacidade dos serviços em responder às necessidades em saúde; acompanhar os efeitos das intervenções; identificar e corrigir problemas; enfim, retroalimentar equipes de saúde, gestores, políticos e comunidades. Não avaliar pode ser comparado a pilotar um avião sem instrumentos de navegação aérea, sem indicadores das condições de voo e do motor. É voar sem bússola, altímetro, velocímetro, indicadores de nível de combustível, óleo e temperatura da água.

Para Felisberto ${ }^{6}$, institucionalizar a avaliação adquire, para nós, um sentido de incorporação à rotina das organizações de saúde, sendo entendida como uma demanda interna de acompanhamento de todo o processo de planejamento e gestão das políticas e/ou programas. O fomento da "cultura avaliativa" requer a qualificação da capacidade técnica, nos diversos níveis do sistema de saúde, viabilizando uma efetiva associação das ações de monitoramento e avaliação como subsidiárias ou intrínsecas ao planejamento e à gestão, dando suporte à formulação de políticas, ao processo decisório e de formação dos sujeitos envolvidos.

Segundo Pisco ${ }^{13}$, a finalidade de políticas de avaliação e de melhoria de qualidade não pode ser apenas para demonstrar os problemas, nem propor soluções para os problemas detectados, mas produzir mudanças apropriadas que conduzam à melhoria da qualidade dos cuidados prestados. O objetivo não é outro senão a identificação, a avaliação e a correção de situações potencialmente melhoráveis.

Contrandiopolus ${ }^{11}$, afirma que a capacidade de institucionalização da avaliação como instrumento para melhorar o sistema de saúde é paradoxal, embora justificada pela suposição de que a informação produzida contribua para racionalizar os processos de decisão. No entanto, observa-se que os diferentes atores que ocupam posições distintas frequentemente não conseguem chegar a um consenso quanto à pertinência dos resultados que a avaliação produz. Retomamos a ideia de que o domínio da tomada de decisão não abrange o da avaliação. Um julgamento realizado por uma instância de decisão deveria considerar, além de os critérios de eficiência, também os que permitissem apreciar as consequências das suas decisões em matéria de equidade de acesso aos serviços e em relação ao respeito às liberdades individuais dos diferentes atores.

Barros et al. ${ }^{17}$, afirma que avaliar tornou-se um ato de maior amplitude e implicação política, e por isso temos tentado anunciar a ideia de que o ato avaliativo compreende um conjunto de práticas ético-políticas, que sustentam intenções e realizam ações com efeito de medir, descrever e julgar o sentido, o mérito e a relevância das intervenções humanas, a fim de produzir aprendizagem para os sujeitos nela envolvidos, de forma particular, e para a sociedade, de forma geral.

Neste sentido, entendemos que para que este processo se institua de forma efetiva, mecanismos pactuados de monitoramento e avaliação da gestão do SUS devem ser implantados em todas as unidades federadas, estabelecendo-se a responsabilização dos estados e municípios e do gestor federal no âmbito do SUS, com vistas ao fortalecimento da capacidade de gestão pública da saúde.

No âmbito da gestão, fica patente que as práticas de monitoramento e avaliação constituemse num carreador de responsabilidades, e de ações rumo a objetivos definidos previamente, onde devem ser previstas decisões em meio a situações diversas e impostas por circunstâncias que os gestores e as equipes que os assessoram muitas vezes não escolhem, sendo necessário a instituição de mecanismos e estratégias que potencializem e consolidem a sua institucionalização.

Sendo assim, concordamos com Tanaka ${ }^{18}$, quando este afirma que se formos capazes de despertar o interesse dos distintos sujeitos sociais em tornar mais racional, por meio da utilização de informações mais objetivas, no cotidiano das escolhas, para a tomada de decisão, estaremos "institucionalizando" a avaliação como um processo extrínseco do cotidiano. A possibilidade de tornar mais objetiva e efetiva as decisões permitirá legitimar o processo de avaliação com os interessados e dessa maneira ganhar espaço e confiança dentro da organização. 
Uma agenda estratégica para

o fortalecimento da institucionalização

dos Processos e Práticas do

Monitoramento e avaliação da gestão do

SUS: É caminhando que se faz o caminho

Como vimos através desta abordagem inicial, temos uma polissemia, polifonia, de temas e termos sobre os aspectos do monitoramento e avaliação da gestão, porém é patente a necessidade da construção de estratégias e de caminhos que propiciem a institucionalização destes processos no âmbito do SUS.

Outro aspecto que fica patente é que o acesso aos resultados do processo de monitoramento e avaliação constitui poderoso instrumento de democratização da informação sobre objetivos, metas e resultados alcançados pelas instâncias de gestão e pelos espaços de controle social, favorecendo o empoderamento e a mobilização social.

Segundo Takeda e Yves ${ }^{16}$, avaliar é uma forma de participação, a da construção e aperfeiçoamento do SUS. É uma oportunidade dos diferentes atores (provedores de serviços em saúde, políticos, gestores, população, universidades e demais instituições de aprendizagem) participarem da configuração que se quer dar ao SUS, sendo ao mesmo tempo um processo de aprendizagem e de controle social.
Tendo em vista estes aspectos, foi organizado um conjunto de iniciativas tendo como base a identificação de evidências que apontavam a necessidade e o grau de cooperação/apoio a ser desenvolvidos junto aos Estados e Municípios por meio das ações do Departamento de Monitoramento e Avaliação da Gestão do SUS (DEMAGS),

Sendo assim, foi definida a construção de uma agenda estratégica como ação imprescindível para a construção de espaços/canais de discussão e diálogo, no sentido da convergência de esforços para a produção de informações estratégicas, de modo a permitir uma visão integrada no que concerne aos aspectos/dimensões da gestão do SUS, e sua correlação com as práticas e processos do monitoramento e avaliação.

A matriz abaixo (Figura 1) traz a sistematização dos projetos estratégicos que o DEMAGS/ SGEP operacionalizou, com base em quatro eixos que foram definidos a partir da leitura de experiências anteriores desenvolvidas pelo Ministério da Saúde, com destaque para o projeto de Avaliação e Melhoria da Qualidade da Atenção Básica, como também pelas reflexões trazidas pelo Grupo de Trabalho de Avaliação da Abrasco.

Optamos por construir uma agenda que permitisse uma abordagem desde os aspectos da pesquisa, passando pelo desenvolvimento e disseminação de tecnologia, e metodologias, até a

Monitoramento e Avaliação da Gestão

Projeto/Processos
Processo e Práticas de M\&A da Gestão

Figura 1. Matriz Lógica utilizada pelo DEMAGS-SGEP para a construção da agenda estratégica de cooperação - Brasil, 2010. 
configuração do processo de apoio e qualificação das equipes gestores estaduais e municipais.

Vale destacar que dentre as ações desenvolvidas, duas questões foram impulsionadoras na construção da agenda, a primeira guardou relação direta com as práticas e os processos do monitoramento e da avaliação da gestão e em particular ao seu processo de institucionalização, o qual foi feito tendo como base os Termos de Compromisso de Gestão (TCG), a segunda teve relação como o processo de monitoramento do indicador relacionado à aprovação do Relatório Anual de Gestão (RAG), durante os anos de 2007 e 2008.

Para ambos os instrumentos, o DEMAGS, à época, utilizava um acompanhamento sistemático dos resultados de adesão ao Pacto pela Saúde para o TGC, por meio do Sistema de Monitoramento do Pacto (SISPACTO), e através dos dados sistematizados pela Comissão Intergestores Tripartite para o RAG.

Em relação ao conjunto de responsabilidades do Pacto de Gestão e para a definição dos critérios que apontaram as responsabilidades que guardam relação direta com os processos de monitoramento e avaliação, foi feita uma leitura do conjunto das responsabilidades existentes nos termos de compromisso de gestão, e a consequente situação de não realização das mesmas, onde se destacaram aquelas referentes as seguintes responsabilidades:

1.8d) desenvolvimento do processo de monitoramento e avaliação, a partir das necessidades de saúde;

4.3) monitorar e fiscalizar a aplicação dos recursos financeiros transferidos aos fundos municipais;

4.4) monitorar o cumprimento pelos municípios dos planos de saúde, dos relatórios de gestão, da operação dos fundos de saúde, indicadores e metas do pacto de gestão, da constituição dos serviços de regulação, de controle, de avaliação e de auditoria e da participação na PPI da atenção à saúde, e;

4.28) elaborar normas complementares para a avaliação tecnológica em saúde.

Com relação ao processo de monitoramento do Relatório Anual de Gestão, nos anos de 2007, 2008 e 2009, verificamos que dos 5564 municípios dentro dos prazos legais, respectivamente, $42 \%$; $48 \%$ e $52 \%$ informaram aprovação nos conselhos de saúde. No tocante aos Estados, para o mesmo período, tivemos respectivamente 19\%; $11 \%$ e $19 \%$, de relatórios aprovados, situação que evidenciava dificuldades na construção do respectivo instrumento, e consequente fragilidade no processo de planejamento.

Esse quadro norteou a construção de um processo de cooperação envolvendo o GT de Avaliação da Abrasco e o Instituto Materno-Infantil de Pernambuco (IMIP), com o objetivo de subsidiar o desenvolvimento de metodologias, trazer para o espaço de discussão atores com envolvimento na produção acadêmica e na pesquisa aplicada, e propiciar a construção das linhas para a qualificação das equipes gestoras em torno de temas voltados para o aprimoramento das práticas e processos do monitoramento e avaliação da gestão.

Cazarin et al. ${ }^{19}$, citando Becker et al., afirmam que este tipo de abordagem favorece a capacidade de gerar novas aprendizagens. Neste âmbito, devem ser envolvidos tanto agentes internos quanto externos à intervenção de forma complementar. As avaliações realizadas em parceria com quem vivencia o problema valoriza o saber local, além de aumentar as chances de que os resultados venham a ser utilizados.

Articulado com os processos anteriores foi constituída uma equipe técnica para organizar o mapeamento das práticas de monitoramento e avaliação desenvolvidas pelos gestores estaduais e municipais, bem como identificar técnicos vinculados a estes processos em âmbito estadual e municipal.

Como iniciativa integradora e articuladora de práticas, o DEMAGS, iniciou a organização de uma rede de caráter interfederativo, denominada RIMA(Rede Interfederativa de Monitoramento e Avaliação), com o objetivo de desenvolver e de difundir processos e práticas metodológicas em monitoramento e avaliação, bem como o ferramentas e tecnologias de apoio à gestão para a tomada de decisão.

Segundo Felisberto et al.$^{20}$, disseminar a cultura avaliativa, tornando essa prática incorporada às rotinas nas três instâncias gestoras do SUS, busca qualificar as ações dos tomadores de decisão (profissionais de saúde, planejadores, gestores e representantes de organizações da sociedade civil), produzindo como efeito, mudanças nesses processos.

$\mathrm{Na}$ perspectiva de reforçar a reflexão sobre o papel da avaliação e a importância de se estabelecer uma nova cultura avaliativa nas diversas esferas da gestão e entre os diferentes atores envolvidos nas práticas de saúde, contribuindo para a institucionalização do monitoramento e da avaliação da gestão do SUS, o componente de quali- 
ficação das equipes gestoras foi contemplado via processo de capacitação. O objetivo deste foi fomentar a produção do conhecimento em monitoramento e avaliação, articulado com a experiência e a prática dos sujeitos envolvidos na gestão do SUS.

Neste ponto, nos valemos das considerações de Travassos $^{21}$, quando destaca que institucionalizar a avaliação requer, além de melhorar a interação entre avaliar e tomar decisões, estratégias e recursos com vistas à produção de conhecimento e o treinamento de pessoal especializado.

Silva e Brandão ${ }^{22}$ apontam para a necessidade de um esforço em aproximar as pessoas da avaliação no sentido de torná-las mais próximas deste objeto, procurando entender o papel da avaliação no processo de desenvolvimento de uma organização enquanto um processo de aprendizagem que pode apoiar pessoas, organizações e grupos sociais a fazer escolhas mais consistentes em relação aos rumos de suas iniciativas.

No que tange a disseminação de informações estratégicas, destacamos a elaboração da publicação do Painel de Indicadores do SUS, que é um instrumento especialmente destinado aos conselheiros de saúde, entidades e movimentos da sociedade civil, as equipes gestores e para utilização na formação acadêmica, com o objetivo de disseminar informações sobre questões relevantes ao Sistema Único de Saúde. Nesse sentido, a elaboração do Painel destaca-se como um dispositivo estratégico que contribui, com o alcance dos efeitos da institucionalização da avaliação e que de forma geral contempla: (i) uso na tomada de decisão; (ii) prestação de contas e (iii) produção de conhecimento ${ }^{23}$.

Com relação ao processo de financiamento a SGEP, vem desde o ano de 2007 descentralizando recursos para Estados e Municípios, no bojo da Política de Gestão Estratégica e Participativa ParticipaSUS. A transferência fundo a fundo para a implementação de ações em monitoramento e avaliação da gestão do SUS, foi feita por meio de atos normativos pactuados na CIT, aportando em quatros anos recursos da ordem de $80 \mathrm{mi}$ lhões de reais.

Sendo assim, nos valemos das reflexões de Felisberto ${ }^{6}$ ao destacar o processo vivenciado pela Coordenação de Acompanhamento e Avaliação do Departamento de Atenção Básica, a partir do ano 2003, o qual desenvolveu diversas ações com vistas à implantação de uma política de avaliação para a atenção básica, calcada em algumas diretrizes: 1) Parcerias com instituições de ensino e pesquisa nacionais e internacionais, bem como com associações profissionais de reconhecido prestigio político e acadêmico; 2) Mobilização de atores institucionais por intermédio de processos amplamente participativos, que buscaram não apenas o estabelecimento de alianças no desenvolvimento de projetos, mas que permitiram, sobretudo, a adesão e a incorporação de propostas de forma mais coerente com as múltiplas necessidades do sistema de saúde; 3) Identificação de fontes de financiamento e garantia de recursos orçamentários para os projetos; 4) Definição de estratégias organizacionais para qualificação de profissionais no campo da avaliação.

Desta forma observamos que as escolhas feitas, os movimentos realizados e as estratégias desenvolvidas guardam coerência com processos já instituídos e que foram, e continuam sendo, muito importantes para a qualificação das práticas de monitoramento e avaliação da gestão no âmbito do SUS.

\section{À guisa de conclusões: Temos limites, mas também temos perspectivas}

Iniciamos nossas considerações finais utilizando as reflexões de Felisberto ${ }^{6}$, que afirma que uma das primeiras convicções explicitadas na condução da gestão é a de que não se deve esperar pelas condições ideais para a superação de problemas estruturais.

Estas considerações nos fazem reforçar a premissa de que a institucionalização das ações de monitoramento e avaliação possuem objetivos intrinsecamente vinculados ao desafio de consolidação do SUS e, como destaca Alves ${ }^{24}$, pode ser entendida, também, como uma intervenção para aumentar a capacidade de governo, com vistas à melhoria de seu projeto político, contribuindo, assim, para a governabilidade do sistema de saúde.

Porém, alguns pontos merecem destaque, e um deles diz respeito à necessidade urgente de que a discussão e, mais do que isto, a operacionalização dos processos que envolvem o monitoramento e a avaliação da gestão sejam valorizadas na agenda dos gestores do SUS, pois segundo Denis e Champagne ${ }^{25}$, os diferentes atores organizacionais podem apoiar a implantação de uma intervenção se virem nela um meio para a atualização de suas estratégias fundamentais.

Sendo assim a iniciativa da SGEP, em cooperar técnica e financeiramente com os gestores estaduais e municipais, teve repercussão no progressivo aumento da apresentação de planos de saúde com a temática da gestão estratégica e participativa, como também pela adesão ao Sistema 
de Apoio ao Relatório Anual de Gestão (SARGSUS), ferramenta eletrônica desenvolvida para apoiar os gestores na construção do RAG estadual e municipal.

Outro aspecto relevante guarda relação com a identificação das inúmeras iniciativas que vem sendo desenvolvidas, tanto no campo acadêmico como no âmbito da gestão, no que concerne a práticas avaliativas, fornecendo pistas capazes de aproximar e de aprimorar estes processos, potencializando-os e consequentemente influindo no aperfeiçoamento da gestão do SUS.

Estas considerações ganham eco nas afirmativas de Bodstein ${ }^{26}$, que destaca o papel que pode desempenhar a avaliação em saúde no sentido de resgatar o necessário compromisso entre a academia e os serviços, entre a pesquisa e a gestão, removendo obstáculos a essa aproximação e contribuindo para o desenvolvimento de uma cultura organizacional no sistema de saúde brasileiro que tenha a avaliação como atividade intrínseca e inseparável do planejamento e da gestão.

Tais afirmativas se conjugam com as considerações de Palos et al. ${ }^{27}$, quando destacam que a construção da capacidade avaliativa como processo de construção de sujeitos está conjugada a um processo pedagógico de perspectiva emancipatória que colabora com o fortalecimento de indivíduos, grupos e organizações.

Porém, para que estas iniciativas surtam efeito, a continuidade do processo de cooperação com o GT de avaliação da ABRASCO e a estraté- gia de construção de uma linha de consenso e discussão com os gestores do SUS, através da articulação com o Conselho Nacional de Secretarias Municipais de Saúde (CONASEMS) e o Conselho Nacional de Secretários Estaduais de Saúde (CONASS), em torno dos processos de aprimoramento do Pacto pela Saúde, por meio da implantação dos dispositivos do Decreto 7508, e a instituição da proposta de uma rede de cooperação e práticas, com certeza deverá ser a estratégia que porá em curso um conjunto de iniciativas capazes de influenciar o fomento do processo de institucionalização da avaliação nos espaços estratégicos da gestão do SUS de caráter tripartite.

Por fim, em que pese o curto espaço de tempo da construção da agenda estratégica, podemos destacar que as recentes mudanças ocorridas no Ministério da Saúde, com o deslocamento das ações do DEMAGS, para a Secretaria Executiva, propiciando o estabelecimento de um processo sistemático de monitoramento e avaliação das prioridades nacionais, a partir do Plano $\mathrm{Na}-$ cional de Saúde, e, consequentemente da implantação das políticas de saúde, nos fazem afirmar que aspectos relevantes da institucionalização das práticas e processos de monitoramento e avaliação, passaram a integrar as operações cotidianas da instância de gestão federal, influindo em seu processo de gestão, norteando o conjunto de responsabilidades inerentes às suas práticas e, consequentemente, à relação tripartite.

\section{Colaboradores}

ALB Carvalho, MF Souza, HE Shimizu, IMVB Senra e KC Oliveira participaram igualmente de todas as etapas de elaboração do artigo. 


\section{Referências}

1. Miranda AS. Proposição de escopo para a avaliação da gestão no Sistema Único de Saúde. Relatório final de consultoria prestada ao Ministério da Saúde. Brasília: Ministério da Saúde (MS); 2005.

2. Santos L, Andrade MOL. SUS: O espaço da Gestão Inovadora e dos Consensos Interfederativos. Aspectos jurídicos, administrativos e financeiros. Campinas: CONASEMS-IDISA; 2007.

3. Paim JS. Avaliação em saúde: uma prática em construção no Brasil. In: Hartz ZMA, Vieira da Silva LM, organizadores. Avaliação em saúde: Dos modelos teóricos à prática na avaliação de Programas e Sistemas de saúde. Rio de Janeiro, Salvador: Editora Fiocruz, EDUFBA; 2005. p. 9-10.

4. Furtado JP, Laperrièrre H. A Avaliação da Avaliação. In: Onocko RC, Furtado JF, organizadores. Desafios da avaliação de programas e serviços em saúde. Campinas: Editora Unicamp; 2011. p. 19-39.

5. Figueiró AC, Frias. PG, Navarro LM. Avaliação em saúde: Conceitos Básicos para as Práticas nas Instituições. In: Samico I, Felisberto E, Figueiró AC, Frias PG, organizadores. Avaliação em Saúde: Bases Conceituais e Operacionais. Rio de Janeiro: Editora Medbook; 2010. p. 1-13.

6. Felisberto E. Da teoria à formulação de uma Política Nacional de Avaliação em Saúde. Cien Saude Colet 2006; 11(3):553-563.

7. Brasil. Ministério da Saúde (MS). Secretaria Executiva. Departamento de Apoio à Descentralização. Nota Técnica: Considerações sobre a instituição de um processo de monitoramento e avaliação do SUS. Brasília: Ministério da Saúde (MS); 2005.

8. Dubois C-A, Champagne F, Bilodeau Histórico da Avaliação. In: Hartz Z, Contandriopoulos AP, Champagne F, Brousselle A, organizadores. Avaliação conceitos e Métodos. Rio de Janeiro: Editora Fiocruz; 2011. p. 19-39.

9. Garcia RC. Subsídio para Organizar Avaliações da Ação Governamental. Brasília: Ministério do Planejamento, Orçamento e Gestão, Instituto de Pesquisa Econômica Aplicada (IPEA); 2001. Texto para Discussão no 776 .

10. Santos EM, Cruz MM, Decotelli PV, Chispin PPM, Abreu DMF. Avaliadores in Situ(ação): reflexões e desafios sobre a institucionalização da prática avaliativa no PN DST/Aids. In: Onocko RC, Furtado JF, organizadores. Desafios da avaliação de programas e serviços em saúde. Campinas: Editora Unicamp; 2011. p. 205-222.

11. Contandriopoulos AP. Avaliando a institucionalização da avaliação. Cien Saude Colet 2006; 11(3):705711.

12. Minayo MCS. O desafio do conhecimento: pesquisa qualitativa em saúde. 9a ed. São Paulo: Hucitec; 2006.

13. Pisco LA: Avaliação como instrumento de mudança. Cien Saude Colet 2006; 11(3):566-568.

14. Brito TA, Jesus CS: Avaliação de serviços de saúde: aspectos conceituais e metodológicos. Revista Digital [periódico na Internet]. $2009 \mathrm{Dez}$ [acessado 2012 mar 14]139. Disponível em: http://www. efdeportes.com/efd139/avaliacao-de-servicos-desaude.htm
15. Hartz MAZ, Vieira LMS, organizadoras. Avaliação em Saúde : Dos Modelos Teóricos à Prática na Avaliação de Programas e Sistemas de Saúde. Salvador, Rio de Janeiro: EDUFBA, Fiocruz; 2005.

16. Takeda S, Yves T. Avaliar, uma responsabilidade. Cien Saude Colet 2006; 11(3):569-571.

17. Barros NF, Castellanos MEP, Motta MC, Alegre SM, Tovey P, Broom A. A produção de sínteses com evidências qualitativas: uma revisão de métodos e técnicas. In: Onocko RC, Furtado JF, organizadores. Desafios da avaliação de programas e serviços em saúde. Campinas: Editora Unicamp; 2011. p. 121-135.

18. Tanaka OY. Caminhos alternativos para a institucionalização da avaliação em saúde. Cien Saude Colet 2006; 11(3):564-576.

19. Cazarin G, Mendes MFM, Albuquerque KM. Perguntas Avaliativas. In: Samico I, Felisberto E, Figueiró AC, Frias PG, organizadores. Avaliação em Saúde: Bases Conceituais e Operacionais. Rio de Janeiro: Editora Medbook; 2010. p. 79-87.

20. Felisberto E, Alves CKA, Bezerra LCA. Institucionalização da Avaliação. In: Samico I, Felisberto E, Figueiró AC, Frias PG, organizadores. Avaliação em Saúde: Bases Conceituais e Operacionais; Rio de Janeiro: Editora Medbook; 2010. p. 143-160.

21. Travassos C. Debate sobre o artigo de Zulmira M.A. Hartz. Cad Saude Publica 1999; 15(2):246-247.

22. Silva RR, Brandão DB. Os Quatro elementos da avaliação. Olho Mágico 2003; 10(2):59-66.

23. Brasil. Ministério da Saúde (MS). Política nacional de monitoramento e avaliação da atenção básica: encontrando caminhos para a institucionalização. Brasília: Ministério da Saúde (MS); 2005.

24. Alves CKA. Institucionalização da avaliação na atenção básica: análise do programa em uma gestão estadual [dissertação]. Recife: Fundação Osvaldo Cruz; 2008.

25. Denis JL, Champange F. Análise de Implantação. In: Hartz ZMA, organizadora. Avaliação em saúde: dos modelos conceituais à prática na análise da implantação de programas. Rio de Janeiro: Editora Fiocruz; 1997.

26. Bodstein R. Atenção Básica na agenda da saúde. Cien Saude Colet 2002; 7(3):401-412.

27. Palos CMC, Brandão DB, Silva RR. Da construção de capacidade avaliatória em iniciativas sociais: algumas reflexões. Ensino-Avaliação e Políticas Públicas em Educação 2005; 13(48):361-374.

Artigo apresentado em 19/12/2011

Aprovado em 10/11/2011

Versão final apresentada em 20/02/2012 